\title{
New topological field theories in two dimensions
}

\author{
R. P. Malik 用 \\ S. N. Bose National Centre for Basic Sciences, \\ Block-JD, Sector-III, Salt Lake, Calcutta-700 098, India
}

\begin{abstract}
It is shown that two $(1+1)$-dimensional (2D) free Abelian- and self-interacting non-Abelian gauge theories (without any interaction with matter fields) belong to a new class of topological field theories. These new theories capture together some of the key features of Witten- and Schwarz type of topological field theories because they are endowed with symmetries that are reminiscent of the Schwarz type theories but their Lagrangian density has the appearance of the Witten type theories. The topological invariants for these theories are computed on a 2D compact manifold and their recursion relations are obtained. These new theories are shown to provide a class of tractable field theoretical models for the Hodge theory in two dimensions of flat (Minkowski) spacetime where there are no propagating degrees of freedom associated with the 2D gauge boson.
\end{abstract}

\footnotetext{
* E-mail: malik@boson.bose.res.in
} 


\section{Introduction}

The history of modern developments in theoretical high energy physics is rich with many cardinal examples which have provided a meeting-ground for theoretical physicists as well as mathematicians. One such example is the subject of topological field theories (TFTs) which has encompassed in its folds such diverse areas of theoretical physics and mathematics as Chern-Simon theories, string theories and matrix models, two-dimensional topological gravity, Morse theory, Donaldson- and Jones polynomials, etc. (see, e.g., Refs. [1-12] and references therein). Broadly speaking, there are two types of TFTs. Witten type TFTs $[2,3]$ are the ones where the quantum action (or the Lagrangian density $\left(\mathcal{L}_{W}\right)$ itself) can be written as the Becchi-Rouet-Stora-Tyutin (BRST) (anti)commutator, i.e.,

$$
\mathcal{L}_{W}=\left\{Q_{B}^{(w)}, V(\Phi, g)\right\}
$$

where $Q_{B}^{(w)}$ is the conserved $\left(\dot{Q}_{B}^{(w)}=0\right)$ and nilpotent $\left(\left(Q_{B}^{(w)}\right)^{2}=0\right)$ BRST charge (in general, metric independent) and $V(\Phi, g)$ is a local expression for the field operators as a function of generic field $\Phi$ and the metric $g$ of spacetime manifold on which the theory is defined. Here the BRST charge is constructed by combining a topological shift symmetry with some kind of local gauge symmetries. In contrast, for the Schwarz type of TFTs [1], the classical action is metric independent and the sum of gauge-fixing and Faddeev-Popov ghost terms are BRST (anti)commutator. In the language of Lagrangian density $\left(\mathcal{L}_{S}\right)$, the Schwarz type of TFTs bear an outlook as

$$
\mathcal{L}_{S}=\mathcal{L}_{C}+\left\{Q_{B}^{(s)}, S(\Phi, g)\right\}
$$

where $\mathcal{L}_{C}$ is the classical metric-independent Lagrangian density that cannot be expressed as the BRST (anti)commutator and $S(\Phi, g)$ is a local field operator that contains all the metric dependence of the theory. For the Schwarz type TFTs, the BRST charge $Q_{B}^{(s)}$ generates only some local gauge symmetries (and there is no presence of a subtle local topological shift symmetry, which is a characteristic feature of Witten type TFTs). It is now obvious that, for both types of TFTs, the symmetric energy momentum tensor is a BRST (anti)commutator. This entails the Hamiltonian density of the theory to be a BRST (anti)commutator. As a consequence, there are no energy excitations in the physical sector of the theory as all the physical states are BRST invariant (i.e. $Q_{B}^{(w, s)} \mid$ phys $>=0$ ). This also ensures that all the correlation functions of the observables (i.e. BRST invariant operators) for the theory are independent of the choice of the metric on the spacetime manifolds.

For the case of a manifold with a trivial flat metric, the TFTs are those theories where there are no propagating (dynamical) degrees of freedom associated with the fields (see, e.g., Ref. [12]). In the present paper, we shall concentrate on the free Abelian- and selfinteracting non-Abelian gauge theories in two-dimensions of spacetime (endowed with a flat Minkowski metric) and show that there are no propagating degrees of freedom associated with the gauge bosons of these theories because of the presence of two nilpotent 
charges which are required to have consistency with the Hodge decomposition theorem (HDT). The symmetries, corresponding to these charges, gauge out the dynamical degrees of freedom of the gauge bosons and the theory becomes topological in nature. In fact, these nilpotent charges will be shown to be analogous to the exterior derivative $d$ $\left(d^{2}=0\right)$ and co-exterior derivative $\delta\left(\delta= \pm * d *, \delta^{2}=0\right)$ of differential geometry which are required in the definition of the HDT which states that, on a compact manifold, any arbitrary $n$-form $f_{n}(n=0,1,2, \ldots)$ can be written as the unique sum of a harmonic form $h_{n}$ $\left(\Delta h_{n}=0, d h_{n}=0, \delta h_{n}=0\right)$, an exact form $\left(d e_{n-1}\right)$ and a co-exact form $\left(\delta c_{n+1}\right)$ as [13-17]

$$
f_{n}=h_{n}+d e_{n-1}+\delta c_{n+1}
$$

where $\Delta=(d+\delta)^{2}=d \delta+\delta d$ is the Laplacian operator. The set of operators $(d, \delta, \Delta)$ is called the de Rham cohomology operators of differential geometry as they define the cohomological properties of a given differential form on a compact manifold.

It has been a long-standing problem to express the de Rham cohomology operators in the language of some local symmetry properties of a given Lagrangian density. Normally, $d\left(d^{2}=0\right)$ operator is identified with the local BRST charge $Q_{B}\left(Q_{B}^{2}=0\right)$ which generates a local, continuous, covariant and nilpotent symmetry transformation for a BRST invariant Lagrangian density corresponding to a given gauge theory. Some very interesting and enlightening attempts [18-21] have been made to express $\delta$ and $\Delta$ for the interacting (non)Abelian gauge theories in arbitrary spacetime dimension but the symmetry transformations turn out to be nonlocal and noncovariant. In the covariant formulation, nilpotency is achieved only for some specific values of the parameters of the theory [22]. Recently, however, it has been shown [23-25] that 2D free Abelian- and self-interacting non-Abelian gauge theories provide a couple of field theoretical models for the Hodge theory where all the de Rham cohomology operators $(d, \delta, \Delta)$ correspond to local and conserved charges which generate local, continuous, covariant and nilpotent (for $d$ and $\delta$ ) symmetries for the BRST invariant Lagrangian density of these theories. In fact, the BRST symmetry (analogue of $d$ ) corresponds to a transformation in which the kinetic energy terms of these theories remain invariant. On the other hand, co-BRST symmetry (analogue of $\delta$ ) is found to be a symmetry transformation under which the gauge-fixing terms remain invariant. The anticommutator of these transformations (analogue of $\Delta$ ) leaves the Faddeev-Popov ghost terms invariant. In these attempts, the topological features of these theories have been very briefly mentioned. The central aim of our present paper is to apply the insights gained in our earlier studies [23-25] to furnish an elaborate proof of the topological nature of these theories as they capture in their realm some very interesting new features. For instance, the form of the Lagrangian density for these theories turns out to be like Witten type TFT but the underlying symmetries are found to be of Schwarz type. Furthermore,

$\dagger$ The one-form $A=A_{\mu} d x^{\mu}$ defines the vector potential for the Abelian gauge theory. The zero-form (gauge-fixing) $\delta A=(\partial \cdot A)$ and the curvature two-form (field strength tensor) $F^{(A)}=d A$ are 'Hodge dual' to each-other in any arbitrary dimension of spacetime. Here $\delta= \pm * d *$ is the co-exterior derivative w.r.t. $d$. The same is not true $\left(F^{(N)} \neq d A\right)$ for the non-Abelian gauge theory (see, e.g., Ref. [14]). 
there are four sets of topological invariants for these theories. These are computed on a 2D compact manifold and energy-momentum tensor is shown to be the sum of a BRST- and co-BRST anticommutator. By exploiting the HDT, it is demonstrated that there are no energy excitations in the physical sector.

The outline of our present paper is as follows.

In section 2, we set up the notations and recapitulate the essentials of our earlier work $[23,24]$ so that the paper can be self-contained. Here we show that the $2 \mathrm{D}$ free Abelian gauge theory is a topological field theory by exploiting the basic ingredients of BRST cohomology and HDT. We demonstrate further that this free theory is also a perfect example of a Hodge theory where, not only the cohomological operators $(d, \delta, \Delta)$ are expressed in terms of generators for some local symmetries, but even the Hodge duality $(*)$ operation is shown to correspond to the existence of a couple of discrete symmetries in the theory. With respect to four conserved and nilpotent charges of the theory, we derive four sets of topological invariants which are shown to be inter-related by the Hodge duality $(*)$ operation and the presence of a discrete symmetry for the ghost action. In fact, the requirement of a specific relationship between the set of topological invariants w.r.t. (anti)BRST- and (anti)coBRST charges, singles out one of the two discrete symmetries of the Lagrangian density which are the analogue of Hodge $(*)$ operation.

Section 3 is devoted to the discussion of a self-interacting 2D non-Abelian gauge theory (without any interaction with matter fields). We derive all the four sets of topological invariants on a 2D compact manifold (w.r.t. all the conserved and nilpotent charges in the theory) and obtain their recursion relations. In analogy with the Abelian gauge theory, we derive a discrete symmetry as an analogue of the Hodge $(*)$ operation for the non-Abelian gauge theory by requiring a certain specific transformation property for the topological invariants of the theory. This discrete symmetry reduces to its Abelian counterpart (as Hodge $(*)$ operation) in the limit when the coupling constant $g$ of non-Abelian gauge theory goes to zero $(g \rightarrow 0)$.

Finally, in section 4, we make some concluding remarks and point out some future directions that can be pursued for further extension of our work.

\section{Abelian gauge theory}

Let us begin with a two $(1+1)$-dimensional $\mathrm{HRST}$ invariant Lagrangian density $\left(\mathcal{L}_{b}\right)$ for the free Abelian gauge theory in the Feynman gauge [26-28]

$$
\begin{gathered}
\mathcal{L}_{b}=-\frac{1}{4} F^{\mu \nu} F_{\mu \nu}-\frac{1}{2}(\partial \cdot A)^{2}-i \partial_{\mu} \bar{C} \partial^{\mu} C \\
\mathcal{L}_{b}=\frac{1}{2} E^{2}-\frac{1}{2}(\partial \cdot A)^{2}-i \partial_{\mu} \bar{C} \partial^{\mu} C
\end{gathered}
$$

\footnotetext{
$\ddagger$ We adopt the notations in which the flat 2D Minkowski metric $\eta_{\mu \nu}=\operatorname{diag}(+1,-1)$ and anti-symmetric Levi-Civita tensor $\varepsilon_{\mu \nu} \varepsilon^{\nu \lambda}=\delta_{\mu}^{\lambda}, \varepsilon^{\mu \nu} \varepsilon_{\mu \nu}=-2 !, \varepsilon_{01}=\varepsilon^{10}=+1, F_{01}=\partial_{0} A_{1}-\partial_{1} A_{0}=E=-\varepsilon^{\mu \nu} \partial_{\mu} A_{\nu}=$ $F^{10}, \square=\eta^{\mu \nu} \partial_{\mu} \partial_{\nu}=\left(\partial_{0}\right)^{2}-\left(\partial_{1}\right)^{2}, \dot{f}=\partial_{0} f$. Note that there is no magnetic component in the 2D field strength tensor $F_{\mu \nu}$. Here Greek indices: $\mu, \nu, \lambda_{\text {.. }}=0,1$ stand for the Minkowski flat spacetime directions.
} 
where $F_{\mu \nu}=\partial_{\mu} A_{\nu}-\partial_{\nu} A_{\mu}$ is the curvature tensor derived from the two-form $F=d A$, $(\partial \cdot A)$ is the gauge-fixing term derived from the zero-form $(\partial \cdot A)=\delta A,(\bar{C}) C$ are the Faddeev-Popov (anti)ghost fields $\left(\bar{C}^{2}=C^{2}=0\right)$ and indices $\mu, \nu=0,1$ represent the flat Minkowski time and space directions. It has been shown $[23,24]$ that the above Lagrangian density remains quasi-invariant (i.e. $\delta_{b} \mathcal{L}_{b}=-\eta \partial_{\mu}\left[(\partial \cdot A) \partial^{\mu} C\right], \delta_{d} \mathcal{L}_{b}=\eta \partial_{\mu}\left[E \partial^{\mu} \bar{C}\right]$ ) under the following on-shell $(\square C=0, \square \bar{C}=0)$ nilpotent $\left(\delta_{b}^{2}=0, \delta_{d}^{2}=0\right) \operatorname{BRST}\left(\delta_{b}\right)$ - and dual $\operatorname{BRST}\left(\delta_{d}\right)$ transformations:

$$
\begin{aligned}
& \delta_{b} A_{\mu}=\eta \partial_{\mu} C \quad \delta_{d} A_{\mu}=-\eta \varepsilon_{\mu \nu} \partial^{\nu} \bar{C} \\
& \begin{array}{ll}
\delta_{b} C=0 & \delta_{d} \bar{C}=0
\end{array} \\
& \delta_{b} \bar{C}=-i \eta(\partial \cdot A) \quad \delta_{d} C=-i \eta E \\
& \delta_{b} E=0 \quad \delta_{d}(\partial \cdot A)=0
\end{aligned}
$$

where $\eta$ is an anticommuting $(\eta C=-C \eta, \eta \bar{C}=-\bar{C} \eta)$ spacetime independent transformation parameter. It will be noticed that, under $\delta_{b}$, it is the electric field $E$ (derived by the application of $d$ on one-form $A=A_{\mu} d x^{\mu}$ ) that remains invariant and the gauge-fixing term transforms (to compensate for the term coming from the variation of ghost term). In contrast, under $\delta_{d}$, it is the gauge-fixing term $(\partial \cdot A)$ (derived from one-form $A=A_{\mu} d x^{\mu}$ by the application of $\delta$ ) that remains invariant and the electric field transforms. The conserved, local and nilpotent $\left(Q_{b}^{2}=0, Q_{d}^{2}=0\right)$ generators for the above transformations are

$$
Q_{b}=\int d x\left[\partial_{0}(\partial \cdot A) C-(\partial \cdot A) \dot{C}\right] \quad Q_{d}=\int d x[E \dot{\bar{C}}-\dot{E} \bar{C}] .
$$

It is very natural to expect that the anticommutator of these two transformations $\left(\left\{\delta_{b}, \delta_{d}\right\}=\right.$ $\left.\delta_{w}\right)$ would also be the symmetry transformation $\left(\delta_{w}\right)$ for the Lagrangian density. This is indeed the case as can be seen that under the following bosonic $\left(\kappa=-i \eta \eta^{\prime}\right)$ transformations

$$
\begin{array}{llc}
\delta_{w} A_{\mu}=\kappa\left(\partial_{\mu} E-\varepsilon_{\mu \nu} \partial^{\nu}(\partial \cdot A)\right) & \delta_{w}(\partial \cdot A)=\kappa \square E \\
\delta_{w} E=\kappa \square(\partial \cdot A) & \delta_{w} C=0 & \delta_{w} \bar{C}=0
\end{array}
$$

the Lagrangian density (2.1) transforms as: $\delta_{w} \mathcal{L}_{b}=\kappa \partial\left[E \partial^{\mu}(\partial \cdot A)-(\partial \cdot A) \partial^{\mu} E\right]$. Here $\eta$ and $\eta^{\prime}$ are the transformation parameters corresponding to $\delta_{b}$ and $\delta_{d}$ respectively. The generator for the above transformation is

$$
W=\int d x\left[\partial_{0}(\partial \cdot A) E-\left(\partial_{0} E\right)(\partial \cdot A)\right] .
$$

The global scale invariance of the Lagrangian density (2.1) under $C \rightarrow e^{-\Sigma} C, \bar{C} \rightarrow$ $e^{\Sigma} \bar{C}, A_{\mu} \rightarrow A_{\mu}$, (where $\Sigma$ is a global parameter), leads to the derivation of a conserved ghost charge $\left(Q_{g}\right)$

$$
Q_{g}=-i \int d x[C \dot{\bar{C}}+\bar{C} \dot{C}] .
$$

Together, these conserved charges satisfy the following algebra [23,24]

$$
\begin{aligned}
& {\left[W, Q_{k}\right]=0 \quad k=g, b, d, a b, a d} \\
& Q_{b}^{2}=Q_{d}^{2}=Q_{a b}^{2}=Q_{a d}^{2}=0 \quad\left\{Q_{d}, Q_{a d}\right\}=0 \\
& \left\{Q_{b}, Q_{d}\right\}=\left\{Q_{a b}, Q_{a d}\right\}=W \quad\left\{Q_{b}, Q_{a b}\right\}=0 \\
& i\left[Q_{g}, Q_{b}\right]=Q_{b} \quad i\left[Q_{g}, Q_{a b}\right]=-Q_{a b} \\
& i\left[Q_{g}, Q_{d}\right]=-Q_{d} \quad i\left[Q_{g}, Q_{a d}\right]=Q_{a d}
\end{aligned}
$$


where $Q_{a b}$ and $Q_{a d}$ are the anti-BRST- and anti-dual BRST charges which can be readily obtained from (2.3) by the replacement $C \rightarrow \pm i \bar{C}, \bar{C} \rightarrow \pm i C$ 3. It can be seen that the ghost number for $Q_{b}$ and $Q_{a d}$ is +1 and that of $Q_{d}$ and $Q_{a b}$ is -1 . Now, given a state $\mid \phi>$ (with ghost number $n$ ) in the quantum Hilbert space (i.e. $i Q_{g}|\phi>=n| \phi>$ ), it can be readily seen, using the above algebra (2.7), that

$$
\begin{array}{lll}
i Q_{g} Q_{b} \mid \phi> & =(n+1) Q_{b} \mid \phi> & i Q_{g} Q_{a d}\left|\phi>=(n+1) Q_{a d}\right| \phi> \\
i Q_{g} Q_{d} \mid \phi> & =(n-1) Q_{d} \mid \phi> & i Q_{g} Q_{a b}\left|\phi>=(n-1) Q_{a b}\right| \phi> \\
i Q_{g} W \mid \phi> & =n W \mid \phi>. &
\end{array}
$$

This shows that the ghost numbers for the states $Q_{b} \mid \phi>\left(\right.$ or $\left.Q_{a d} \mid \phi>\right), Q_{d} \mid \phi>\left(\right.$ or $\left.Q_{a b} \mid \phi>\right)$ and $W \mid \phi>$ in the quantum Hilbert space are $(n+1),(n-1)$ and $n$ respectively. As far as underlying algebra is concerned, the above symmetry generators $Q_{b}, Q_{d}$ and $W$ obey the same kind of algebra as their counterparts (de Rham cohomology operators $d, \delta$ and $\Delta$ ) in differential geometry. The latter algebra can be succinctly expressed as

$$
\begin{array}{lcc}
d^{2}=0 & \delta^{2}=0 & \Delta=(d+\delta)^{2}=d \delta+\delta d \\
{[\Delta, d]=0} & {[\Delta, \delta]=0} & \Delta=\{d, \delta\} \neq 0
\end{array}
$$

It is a peculiarity of the BRST formalism that the above cohomological operators can be also identified with the generators $Q_{a d}, Q_{a b}$ and $W=\left\{Q_{a b}, Q_{a d}\right\}$ respectively. Thus, the mapping is: $\left(Q_{b}, Q_{a d}\right) \Leftrightarrow d,\left(Q_{d}, Q_{a b}\right) \Leftrightarrow \delta, W=\left\{Q_{b}, Q_{d}\right\}=\left\{Q_{a d}, Q_{a b}\right\} \Leftrightarrow \Delta$. This analogy enables us to express the Hodge decomposition theorem in the quantum Hilbert space of states where any arbitrary state $\mid \phi>_{n}$ (with ghost number $n$ ) can be written as the sum of a harmonic state $\mid \omega>_{n}\left(W\left|\omega>_{n}=0, Q_{b}\right| \omega>_{n}=0, Q_{d} \mid \omega>_{n}=0\right)$, a BRST exact state $\left(Q_{b} \mid \theta>_{n-1}\right)$ and a co-BRST exact state $\left(Q_{d} \mid \chi>_{n+1}\right)$. Mathematically, this statement (which is the analogue of eqn. (1.3)) can be expressed, in two equivalent ways, as

$$
\left|\phi>_{n}=\right| \omega>_{n}+Q_{b}\left|\theta>_{n-1}+Q_{d}\right| \chi>_{n+1} \equiv\left|\omega>_{n}+Q_{a d}\right| \theta>_{n-1}+Q_{a b} \mid \chi>_{n+1} .
$$

It is worth pointing out that the sets of charges $\left(Q_{b}, Q_{d}\right)$ and $\left.\left(Q_{a b}, Q_{a d}\right)\right)$ are the dual sets, because $Q_{b}$ and $Q_{d}$ are dual to each-other as are $Q_{a b}$ and $Q_{a d}$. To elaborate this claim, it can be seen that, under the following separate and independent transformations

$$
\begin{gathered}
C \rightarrow \pm i \bar{C} \quad \bar{C} \rightarrow \pm i C \quad A_{\mu} \rightarrow A_{\mu} \quad \partial_{\mu} \rightarrow \pm i \varepsilon_{\mu \nu} \partial^{\nu} \\
C \rightarrow \pm i \bar{C} \quad \bar{C} \rightarrow \pm i C \quad A_{\mu} \rightarrow \mp i \varepsilon_{\mu \nu} A^{\nu}
\end{gathered}
$$

the two-form (electric) field $E$ and the zero-form (gauge-fixing) field $(\partial \cdot A)$ are related with each-other as: $E \rightarrow \pm i(\partial \cdot A),(\partial \cdot A) \rightarrow \pm i E$. Thus, we see that under the above transformations: (i) the Lagrangian density (2.1) remains invariant. (ii) The dual BRST

$\S$ Note that the discrete transformations: $C \rightarrow \pm i \bar{C}, \bar{C} \rightarrow \pm i C$ are the symmetry transformations for the ghost action $\left(I_{F . P .}=-i \int d^{D} x \partial_{\mu} \bar{C} \partial^{\mu} C\right)$ in any arbitrary dimension of spacetime. 
symmetry transformations $\delta_{d}$ can be obtained from the BRST transformations $\delta_{b}$ in (2.2). (iii) The symmetry generators $Q_{k},(k=b, a b, d, a d, g)$ and $W$ transform as:

$$
\begin{aligned}
& Q_{b} \rightarrow Q_{d} \quad Q_{d} \rightarrow Q_{b} \quad Q_{a b} \rightarrow Q_{a d} \\
& Q_{a d} \rightarrow Q_{a b} \quad Q_{g} \rightarrow-Q_{g} \quad W \rightarrow W .
\end{aligned}
$$

(iv) The algebraic structure of (2.7) remains form-invariant under (2.12).

The transformations (2.10) and (2.11) are the analogue of the Hodge $(*)$ operation of differential geometry. To clarify this assertion, first, we note the consequences of two successive operations of $(*)$ on the generic field $\Phi$ of the theory, namely;

$$
*(* \Phi)= \pm \Phi
$$

Here $*$ operation corresponds to transformations $(2.10)$ and $(+)$ sign stands for $\Phi=A_{\mu}$ and (-) sign for $\Phi=C, \bar{C}, E,(\partial \cdot A)$. Under transformations (2.11), the analogue of (2.13) is $*(* \Phi)=-\Phi$ for all the fields of the theory (i.e. $\Phi=A_{\mu}, C, \bar{C}, E,(\partial \cdot A)$. Now, it is straightforward to check that $\delta_{d}$ and $\delta_{b}$ are related to each other as

$$
\delta_{d} \Phi= \pm * \delta_{b} * \Phi
$$

where $\delta_{d}$ and $\delta_{b}$ are the nilpotent transformations in $(2.2)$ and the $(*)$ operation corresponds to transformations in (2.10). The signs in (2.14) are governed by the corresponding signatures in (2.13). For the $(*)$ operation corresponding to (2.11), the analogue of $(2.14)$, is

$$
\delta_{d} \Phi=-* \delta_{b} * \Phi
$$

for the generic field $\Phi=A_{\mu}, C, \bar{C}, E,(\partial \cdot A)$. It is obvious that the relation between nilpotent transformations $\delta_{d}$ and $\delta_{b}$, acting on a generic field $\Phi$, is same as the relation between dual exterior derivative $\delta(= \pm * d *)$ and exterior derivative $d$ acting on a differential form defined on a compact manifold. It will be noticed that duality transformations in $2 \mathrm{D}$ and $4 \mathrm{D}$ are different [29-31]. This is the reason that, for the 4D $(3+1)$ theories, it has been shown [32] that under $*$ operation: $Q_{b} \rightarrow Q_{d}, Q_{d} \rightarrow-Q_{b}$ which is like the electromagnetic duality transformations for the Maxwell equations where: $\mathbf{E} \rightarrow \mathbf{B}, \mathbf{B} \rightarrow-\mathbf{E}$. In fact, it is due to the peculiarity of duality transformations in $2 \mathrm{D}$ that a reverse relation also exist which allows one to express $\delta_{b}$ in terms of $\delta_{d}$ as

$$
\delta_{b} \Phi= \pm * \delta_{d} * \Phi \quad \text { and } \quad \delta_{b} \Phi=-* \delta_{d} * \Phi
$$

corresponding to transformations (2.10) and (2.11), respectively.

Exploiting the fact that conserved charges $Q_{r}(r=b, d, a b, a d)$ are the generators for the transformations $\delta_{r} \Phi=-i \eta\left[\Phi, Q_{r}\right]_{ \pm}$where $(+)-$stand for the (anti)commutator corresponding to $\Phi$ being (fermionic)bosonic in nature, it can be readily seen that the Lagrangian density in (2.1) can be written, modulo some total derivatives, as

$$
\mathcal{L}_{b}=\left\{Q_{d}, T_{1}\right\}+\left\{Q_{b}, T_{2}\right\} \equiv\left\{Q_{a d}, P_{1}\right\}+\left\{Q_{a b}, P_{2}\right\}
$$


where $T_{1}=\frac{1}{2} E C, T_{2}=-\frac{1}{2}(\partial \cdot A) \bar{C}, P_{1}=\frac{i}{2} E \bar{C}, P_{2}=-\frac{i}{2}(\partial \cdot A) C$. Furthermore, using the on-shell nilpotent symmetries of (2.2), it can be checked that the above Lagrangian density can be re-expressed as a sum of the (anti)BRST- and (anti)-dual BRST invariant parts and a total derivative, as

$$
\eta \mathcal{L}_{b}=\delta_{d}\left(i T_{1}\right)+\delta_{b}\left(i T_{2}\right)+\eta \partial_{\mu} Y^{\mu} \equiv \delta_{a d}\left(i P_{1}\right)+\delta_{a b}\left(i P_{2}\right)+\eta \partial_{\mu} Y^{\mu}
$$

where the nilpotent transformations $\delta_{a b}$ and $\delta_{a d}$ can be readily derived from (2.2) by exploiting the substitution: $C \rightarrow i \bar{C}, \bar{C} \rightarrow i C$ and $Y^{\mu}=\frac{i}{2}\left(\bar{C} \partial^{\mu} C+\partial^{\mu} \bar{C} C\right)$. The appearance of the Lagrangian density (2.1) (in the form (2.17)) is reminiscent of the Witten-type topological field theories (1.1) where it is possible to express the Lagrangian density of a TFT as a BRST (anti)commutator. Even though in our case, we have two sets of nilpotent charges $\left(Q_{b}, Q_{d}\right)$ as well as $\left(Q_{a b}, Q_{a d}\right)$, the outlook of the Lagrangian density (2.17) is same as the Witten type TFTs because the physical states are the harmonic states (of the Hodge decomposition theorem) which satisfy $Q_{(b, a b)} \mid$ phys $>=0, Q_{(d, a d)} \mid$ phys $>=0$. It should be noted that the appearance in (2.17), is completely different from the Schwarz type of theories where the Lagrangian density (1.2) is a sum of a BRST (anti)commutator and a piece that can never be expressed as a BRST (anti)commutator [1,12]. At this stage, however, we note that we have only local gauge type symmetries and there is no trace of any topological shift symmetries. Hence, it is clear that, from the symmetry point of view, the free 2D $U(1)$ gauge theory is like Schwarz type topological theories.

One of the key properties of TFTs is the absence of any energy excitations in the theory. This aspect is governed by the expression for the symmetric energy-momentum tensor $\left(T_{\alpha \beta}^{(s)}\right)$. It is interesting to check that the expression for this symmetric tensor for the generic field $\Phi=A_{\mu}, C, \bar{C}$, present in the Lagrangian density (2.1), is

$$
\begin{aligned}
T_{\alpha \beta}^{(s)} & =\frac{1}{2} \partial_{\alpha} \Phi \frac{\partial \mathcal{L}_{b}}{\partial_{\beta} \Phi}+\frac{1}{2} \partial_{\beta} \Phi \frac{\partial \mathcal{L}_{b}}{\partial_{\alpha} \Phi}-\eta_{\alpha \beta} \mathcal{L}_{b} \\
& \equiv-\frac{1}{2}\left[\varepsilon_{\alpha \rho} E+\eta_{\alpha \rho}(\partial \cdot A)\right] \partial_{\beta} A^{\rho}-\frac{1}{2}\left[\varepsilon_{\beta \rho} E+\eta_{\beta \rho}(\partial \cdot A)\right] \partial_{\alpha} A^{\rho} \\
& -i \partial_{\alpha} \bar{C} \partial_{\beta} C-i \partial_{\beta} \bar{C} \partial_{\alpha} C-\eta_{\alpha \beta} \mathcal{L}_{b} .
\end{aligned}
$$

This equation, with the use of (2.17), can be explicitly expressed as

$$
\begin{aligned}
T_{\alpha \beta}^{(s)} & =\left\{Q_{b}, V_{\alpha \beta}^{(1)}\right\}+\left\{Q_{d}, V_{\alpha \beta}^{(2)}\right\} \\
V_{\alpha \beta}^{(1)} & =\frac{1}{2}\left[\left(\partial_{\alpha} \bar{C}\right) A_{\beta}+\left(\partial_{\beta} \bar{C}\right) A_{\alpha}+\eta_{\alpha \beta}(\partial \cdot A) \bar{C}\right] \\
V_{\alpha \beta}^{(2)} & =\frac{1}{2}\left[\left(\partial_{\alpha} C\right) \varepsilon_{\beta \rho} A^{\rho}+\left(\partial_{\beta} C\right) \varepsilon_{\alpha \rho} A^{\rho}-\eta_{\alpha \beta} E C\right] .
\end{aligned}
$$

This shows that, when the Hamiltonian density $\hat{T}_{00}^{(s)}$ is sandwiched between two physical states (i.e. $<p h y s\left|\hat{T}_{00}^{(s)}\right| p h y s^{\prime}>=0$ ) it turns out to be zero because hermitian operators $Q_{b}, Q_{d}$ annihilate the harmonic states (which are the BRST- and co-BRST invariant physical

`Here, and in what follows, we shall be exploiting only nilpotent charges $Q_{b}$ and $Q_{d}$ for our purposes. However, $Q_{a b}$ and $Q_{a d}$ could be used equally well for the same objectives. All one has to do is to exploit the substitution: $C \rightarrow \pm i \bar{C}, \bar{C} \rightarrow \pm i C$ judiciously. 
states $\left(Q_{b} \mid\right.$ phys $>=0, Q_{d} \mid$ phys $\left.>=0\right)$ in the theory). In fact, conditions $Q_{b} \mid$ phys $>=$ $0, Q_{d} \mid$ phys $>=0$ imply that $(\partial \cdot A) \mid$ phys $>=0, \varepsilon^{\mu \nu} \partial_{\mu} A_{\nu} \mid$ phys $>=0$, respectively [23,24]. This ensures that there are no propagating degrees of freedom in the theory as both the components $A_{0}$ and $A_{1}$ of a $2 \mathrm{D}$ photon are conserved quantities (w.r.t. time). In other words, there is no evolution in the system w.r.t. the evolution (time) parameter of the theory. This condition confirms the topological nature of the free $2 \mathrm{D} U(1)$ gauge theory in the flat Minkowski spacetime.

The topological nature is further confirmed by the existence of two sets of topological invariants w.r.t. conserved and on-shell $(\square C=\square \bar{C}=0)$ nilpotent $\left(Q_{b}^{2}=0, Q_{d}^{2}=0\right)$ BRST- and co-BRST charges. For the 2D compact manifold, these are

$$
I_{k}=\oint_{C_{k}} V_{k} \quad J_{k}=\oint_{C_{k}} W_{k} \quad(k=0,1,2)
$$

where $C_{k}$ are the $k$-dimensional homology cycles in the $2 \mathrm{D}$ manifold and $V_{k}$ and $W_{k}$ are the $k$-forms. These forms, w.r.t. the BRST charge $Q_{b}$, are

$$
\begin{aligned}
& V_{0}=-(\partial \cdot A) C \quad V_{1}=\left[-(\partial \cdot A) A_{\mu}+i C \partial_{\mu} \bar{C}\right] d x^{\mu} \\
& V_{2}=i\left[A_{\mu} \partial_{\nu} \bar{C}-\frac{\bar{C}}{2} F_{\mu \nu}\right] d x^{\mu} \wedge d x^{\nu}
\end{aligned}
$$

and the same, w.r.t. the dual BRST charge $Q_{d}$, are

$$
\begin{aligned}
& W_{0}=E \bar{C} \quad W_{1}=\left[\bar{C} \varepsilon_{\mu \rho} \partial^{\rho} C-i E A_{\mu}\right] d x^{\mu} \\
& W_{2}=i\left[\varepsilon_{\mu \rho} \partial^{\rho} C A_{\nu}+\frac{C}{2} \varepsilon_{\mu \nu}(\partial \cdot A)\right] d x^{\mu} \wedge d x^{\nu}
\end{aligned}
$$

It will be noticed here that there are two more sets of topological invariants $\left(\tilde{V}_{k}, \tilde{W}_{k}\right)$ w.r.t. the conserved and on-shell $(\square C=\square \bar{C}=0)$ nilpotent $\left(Q_{a b}^{2}=Q_{a d}^{2}=0\right)$ anti-BRST- and anti-dual BRST charges. These can be derived from (2.21) and (2.22) by the substitution: $C \rightarrow i \bar{C}, \bar{C} \rightarrow i C$. For $k=1,2$, all these four invariants obey a specific recursion relation

$$
\begin{array}{lcrr}
\delta_{b} V_{k}=\eta d V_{k-1} & \delta_{a b} \tilde{V}_{k}=\eta d \tilde{V}_{k-1} & d=d x^{\mu} \partial_{\mu} \\
\delta_{d} W_{k}=\eta \delta W_{k-1} & \delta_{a d} \tilde{W}_{k}=\eta \delta \tilde{W}_{k-1} & \delta=i d x^{\mu} \varepsilon_{\mu \nu} \partial^{\nu}
\end{array}
$$

which is a typical feature for the existence of any TFTs. It is very interesting to note that, under $(*)$ operation corresponding to transformations in $(2.10)$, the above topological invariants, for $k=0,1,2$, transform as

$$
V_{k} \rightarrow W_{k} \quad \tilde{V}_{k} \rightarrow \tilde{W}_{k} \quad W_{k} \rightarrow(-1)^{k} V_{k} \quad \tilde{W}_{k} \rightarrow(-1)^{k} \tilde{V}_{k} .
$$

Mathematically, this statement can be succinctly expressed as

$$
* V_{k}=W_{k} \quad * \tilde{V}_{k}=\tilde{W}_{k} \quad *\left(* V_{k}\right)=(-1)^{k} V_{k} \quad *\left(* \tilde{V}_{k}\right)=(-1)^{k} \tilde{V}_{k}
$$

where $*$ operation corresponds to transformations in (2.10) and $k=0,1,2$ stands for the degree of the forms on the $2 \mathrm{D}$ compact manifold. Another interesting point to be noted is the fact that the requirement: $I_{k} \rightarrow J_{k}$ under $*$ operation, singles out transformations 
(2.10) from (2.10) and (2.11) which are symmetry transformations for the Lagrangian density (2.1). This assertion will play an important role in the discussion of topological invariants and their transformations under $*$ operation for the case of non-Abelian gauge theory (see, e.g., Sec. 3 below).

To conclude this section, we note that $2 \mathrm{D}$ free $U(1)$ gauge theory is a prototype example of a field theoretical model for the Hodge theory. This theory also turns out to be a new type of topological field theory. The form of its Lagrangian density looks like Witten type of TFTs but its symmetries are just like that of Schwarz type TFTs (as there is a conspicuous absence of the topological shift symmetry in the theory).

\section{Non-Abelian gauge theory}

Let us start off with the $(1+1)$-dimensional BRST invariant Lagrangian density $\left(\mathcal{L}_{B}\right)$ for the self-interacting non-Abelian gauge theory in the Feynman gauge [26-28]

$$
\begin{aligned}
\mathcal{L}_{B} & =-\frac{1}{4} F^{\mu \nu a} F_{\mu \nu}^{a}-\frac{1}{2}(\partial \cdot A)^{a}(\partial \cdot A)^{a}-i \partial_{\mu} \bar{C}^{a} D^{\mu} C^{a} \\
& =\frac{1}{2} E^{a} E^{a}-\frac{1}{2}(\partial \cdot A)^{a}(\partial \cdot A)^{a}-i \partial_{\mu} \bar{C}^{a} D^{\mu} C^{a}
\end{aligned}
$$

where $F_{01}^{a}=\partial_{0} A_{1}^{a}-\partial_{1} A_{0}^{a}+g f^{a b c} A_{0}^{b} A_{1}^{c}=E^{a}$ is the "coloured" (group-valued) electric field derived from the "coloured" gauge connections $A_{0}^{a}$ and $A_{1}^{a},\left(\bar{C}^{a}\right) C^{a}$ are the anticommuting Faddeev-Popov (anti)ghost fields $\left(\left(\bar{C}^{a}\right)^{2}=\left(C^{a}\right)^{2}=0\right)$, the covariant derivative is: $D_{\mu} C^{a}=$ $\partial_{\mu} C^{a}+g f^{a b c} A_{\mu}^{b} C^{c}$, spacetime indices are: $\mu, \nu, \lambda \ldots \ldots=0,1$ and group indices $a, b, c \ldots=$ $1,2,3 \ldots$ correspond to a compact Lie group, $g$ is the coupling constant denoting the strength of the interaction amongst gauge fields and structure constants $f^{a b c}$ are chosen to be totally antisymmetric for the above compact Lie algebra [33]. It has been demonstrated [25] that the above Lagrangian density remains quasi-invariant $\left(\delta_{B} \mathcal{L}_{B}=-\eta \partial_{\mu}\left[(\partial \cdot A)^{a} D^{\mu} c^{a}\right], \delta_{D} \mathcal{L}_{B}=\right.$ $\left.\eta \partial_{\mu}\left[E^{a} \partial^{\mu} \bar{C}^{a}\right]\right)$ under the following on-shell $\left(\partial_{\mu} D^{\mu} C^{a}=0, D_{\mu} \partial^{\mu} \bar{C}^{a}=0\right)$ nilpotent $\left(\delta_{B}^{2}=\right.$ $\left.\delta_{D}^{2}=0\right)$ BRST- and dual BRST transformations

$$
\begin{aligned}
& \delta_{B} A_{\mu}^{a} \quad=\eta D_{\mu} C^{a} \quad \delta_{D} A_{\mu}^{a}=-\eta \varepsilon_{\mu \nu} \partial^{\nu} \bar{C}^{a} \\
& \delta_{B} C^{a} \quad=-\frac{\eta g}{2} f^{a b c} C^{b} C^{c} \quad \delta_{D} \bar{C}^{a}=0 \\
& \delta_{B} \bar{C}^{a} \quad=-i \eta(\partial \cdot A)^{a} \quad \delta_{D} C^{a}=-i \eta E^{a} \\
& \delta_{B} E^{a} \quad=\eta g f^{a b c} E^{b} C^{c} \quad \delta_{D}(\partial \cdot A)^{a}=0 \\
& \delta_{B}(\partial \cdot A)^{a}=\eta \partial_{\mu} D^{\mu} C^{a} \quad \delta_{D} E^{a}=\eta D_{\mu} \partial^{\mu} \bar{C}^{a}
\end{aligned}
$$

where $\eta$ is an anticommuting $\left(\eta C^{a}=-C^{a} \eta, \eta \bar{C}^{a}=-\bar{C}^{a} \eta\right)$ spacetime independent transformation parameter. It is quite straightforward to check that the anticommutator of the above transformations generate a bosonic $\left(\kappa=-i \eta \eta^{\prime}\right)$ symmetry transformation $\left(\delta_{W}=\left\{\delta_{B}, \delta_{D}\right\}\right)$

$$
\begin{array}{ll}
\delta_{W} A_{\mu}^{a} & =\kappa\left[D_{\mu} E^{a}-\varepsilon_{\mu \nu} \partial^{\nu}(\partial \cdot A)^{a}-i g f^{a b c} \varepsilon_{\mu \nu} \partial^{\nu} \bar{C}^{b} C^{c}\right] \\
\delta_{W}(\partial \cdot A)^{a} & =\kappa\left[\partial_{\mu} D^{\mu} E^{a}+i g f^{a b c} \varepsilon^{\mu \nu} \partial_{\mu} \bar{C}^{b} \partial_{\nu} C^{c}\right] \\
\delta_{W} E^{a} & =\kappa\left[D_{\mu} \partial^{\mu}(\partial \cdot A)^{a}-\varepsilon^{\mu \nu} D_{\mu} D_{\nu} E^{a}+i g f^{a b c} D_{\mu}\left(\partial^{\mu} \bar{C}^{b} C^{c}\right)\right] \\
\delta_{W} C^{a} & =0 \quad \delta_{W} \bar{C}^{a}=0
\end{array}
$$


as the Lagrangian density $\mathcal{L}_{B}$ transforms to a total derivative [25]

$$
\begin{aligned}
\delta_{W} \mathcal{L}_{B} & =\kappa \partial_{\mu}\left[Z^{\mu}\right] \\
Z^{\mu} & =E^{a} \partial^{\mu}(\partial \cdot A)^{a}-(\partial \cdot A)^{a} D^{\mu} E^{a}+i g f^{a b c}\left(E^{a} \partial^{\mu} \bar{C}^{b}-\varepsilon^{\mu \nu} \partial_{\nu} \bar{C}^{a}(\partial \cdot A)^{b}\right) C^{c}
\end{aligned}
$$

This bosonic symmetry transformation and the nilpotent symmetry transformations in (3.2) are generated by the conserved charges

$$
\begin{aligned}
W^{(N)} & =\int d x\left[(\partial \cdot A)^{a} D_{0} E^{a}-E^{a} \partial_{0}(\partial \cdot A)^{a}-i g f^{a b c}\left(E^{a} \dot{C}^{a}+\partial_{1} \bar{C}^{a}(\partial \cdot A)^{b}\right) C^{c}\right] \\
Q_{B} & =\int d x\left[\partial_{0}(\partial \cdot A)^{a} C^{a}-(\partial \cdot A)^{a} D_{0} C^{a}+\frac{i g}{2} f^{a b c} \dot{C}^{a} C^{b} C^{c}\right] \\
Q_{D} & =\int d x\left[E^{a} \dot{\bar{C}}^{a}-D_{0} E^{a} \bar{C}^{a}-i g f^{a b c} \bar{C}^{a} \partial_{1} \bar{C}^{b} C^{c}\right] .
\end{aligned}
$$

The continuous global scale invariance of the Lagrangian density (3.1) under transformations $C^{a} \rightarrow e^{-\Lambda} C^{a}, \bar{C}^{a} \rightarrow e^{\Lambda} \bar{C}^{a}, A_{\mu}^{a} \rightarrow A_{\mu}^{a}$ (where $\Lambda$ is a global parameter) leads to the derivation of a conserved ghost charge $\left(Q_{G}\right)$

$$
Q_{G}=-i \int d x\left[C^{a} \partial_{0} \bar{C}^{a}+\bar{C}^{a} D_{0} C^{a}\right]
$$

Together these generators obey the following extended BRST algebra

$$
\begin{aligned}
& Q_{B}^{2}=\frac{1}{2}\left\{Q_{B}, Q_{B}\right\}=0 \quad Q_{D}^{2}=\frac{1}{2}\left\{Q_{D}, Q_{D}\right\}=0 \\
& \left\{Q_{B}, Q_{D}\right\}=W^{(N)} \quad\left[W^{(N)}, Q_{k}\right]=0 \\
& i\left[Q_{G}, Q_{B}\right]=Q_{B} \quad i\left[Q_{G}, Q_{D}\right]=-Q_{D} .
\end{aligned}
$$

This algebra is reminiscent of the algebra obeyed by the de Rham cohomology operators $d, \delta, \Delta$ as given in (2.9a). As a consequence of this algebra, it is clear that, given a state $\mid \psi>_{n}$ with ghost number $n$ (i.e. $i Q_{G}\left|\psi>_{n}=n\right| \psi>_{n}$ ) in the quantum Hilbert space of states, the following relations are correct

$$
i Q_{G} Q_{(B, D)}\left|\psi>_{n}=(n \pm 1) Q_{(B, D)}\right| \psi>_{n} \quad i Q_{G} W^{(N)}\left|\psi>_{n}=n W^{(N)}\right| \psi>_{n} .
$$

This demonstrates that the ghost numbers for the states $Q_{B}\left|\psi>_{n}, Q_{D}\right| \psi>_{n}$ and $W^{(N)} \mid \psi>_{n}$ are $(n+1),(n-1)$ and $n$ respectively. This is analogous to the change in the degree of the form $f_{n}$, when operated upon by the cohomological operators $d, \delta, \Delta$ defined on a compact manifold. It is now obvious that the Hodge decomposition theorem (1.3) can be implemented in the quantum Hilbert space of states $\left|\psi>_{n}=\right| \tilde{\omega}>_{n}+Q_{B}\left|\tilde{\theta}>_{n-1}+Q_{D}\right| \tilde{\chi}>_{n+1}$ (which is the analogue of $(2.9 \mathrm{~b})$ for the non-Abelian case).

It is well-known that, for the Witten type TFTs, the Lagrangian density (or the action itself) is a BRST (anti)commutator (1.1). With this as a backdrop, it can be noticed that, modulo some total derivatives, the Lagrangian density (3.1) can be recast as the sum of a BRST- and co-BRST anticommutator (or a BRST and co-BRST invariant parts)

$$
\begin{aligned}
& \mathcal{L}_{B}=\left\{Q_{D}, S_{1}\right\}+\left\{Q_{B}, S_{2}\right\} \\
& \eta \mathcal{L}_{B}=\delta_{D}\left(i S_{1}\right)+\delta_{B}\left(i S_{2}\right) \quad S_{1}=\frac{1}{2} E^{a} C^{a} \quad S_{2}=-\frac{1}{2}(\partial \cdot A)^{a} \bar{C}^{a}
\end{aligned}
$$


More precisely, the above expression can be seen to produce

$$
\begin{aligned}
\mathcal{L}_{B} & =\frac{1}{2} E^{a} E^{a}-\frac{1}{2}(\partial \cdot A)^{a}(\partial \cdot A)^{a}-i \partial_{\mu} \bar{C}^{a} D^{\mu} C^{a}+\partial_{\mu}\left[X^{\mu}\right] \\
X^{\mu} & =\frac{i}{2}\left(\bar{C}^{a} D^{\mu} C^{a}+\partial^{\mu} \bar{C}^{a} C^{a}\right) .
\end{aligned}
$$

This shows that, with two nilpotent charges $Q_{B}$ and $Q_{D}$, the Lagrangian density (3.1) resembles with that of the Witten type TFTs if we choose the physical states as BRST- and co-BRST invariant (harmonic state) in the Hodge decomposition theorem (i.e. $Q_{B} \mid$ phys $>=$ $0, Q_{D} \mid$ phys $\left.>=0\right)$. It will be noticed, however, that in our discussions of the symmetries for the theory, we do not have any topological shift symmetry. Thus, from the symmetry angle, 2D self-interacting non-Abelian gauge theory is like Schwarz type TFTs which possess only local gauge symmetries.

The expression for the symmetric energy-momentum tensor $\left(\tilde{T}_{\mu \nu}^{(s)}\right)$ for the Lagrangian density (3.1) is

$$
\begin{aligned}
\tilde{T}_{\mu \nu}^{(s)} & =-\frac{1}{2}\left[\varepsilon_{\mu \rho} E^{a}+\eta_{\mu \rho}(\partial \cdot A)^{a}\right] \partial_{\nu} A^{\rho a}-\frac{1}{2}\left[\varepsilon_{\nu \rho} E^{a}+\eta_{\nu \rho}(\partial \cdot A)^{a}\right] \partial_{\mu} A^{\rho a} \\
& -\frac{i}{2}\left(\partial_{\mu} \bar{C}^{a}\right)\left(\partial_{\nu} C^{a}+D_{\nu} C^{a}\right)-\frac{i}{2}\left(\partial_{\nu} \bar{C}^{a}\right)\left(\partial_{\mu} C^{a}+D_{\mu} C^{a}\right)-\eta_{\mu \nu} \mathcal{L}_{B} .
\end{aligned}
$$

Here $\mathcal{L}_{B}$ is the Lagrangian in (3.1) (or equivalently (3.9)). This expression can be re-written, modulo some total derivatives, as the sum of BRST- and co-BRST (anti)commutators (or, equivalently, as the BRST- and co-BRST invariant parts)

$$
\begin{aligned}
& \tilde{T}_{\mu \nu}^{(s)}=\left\{Q_{B}, L_{\mu \nu}^{(1)}\right\}+\left\{Q_{D}, L_{\mu \nu}^{(2)}\right\} \quad \eta \tilde{T}_{\mu \nu}^{(s)}=\delta_{B}\left(i L_{\mu \nu}^{(1)}\right)+\delta_{D}\left(i L_{\mu \nu}^{(2)}\right) \\
& L_{\mu \nu}^{(1)}=\frac{1}{2}\left[\left(\partial_{\mu} \bar{C}^{a}\right) A_{\nu}^{a}+\left(\partial_{\nu} \bar{C}^{a}\right) A_{\mu}^{a}+\eta_{\mu \nu}(\partial \cdot A)^{a} \bar{C}^{a}\right] \\
& L_{\mu \nu}^{(2)}=\frac{1}{2}\left[\left(\partial_{\mu} C^{a}\right) \varepsilon_{\nu \rho} A^{\rho a}+\left(\partial_{\nu} C^{a}\right) \varepsilon_{\nu \rho} A^{\rho a}-\eta_{\mu \nu} E^{a} C^{a}\right] .
\end{aligned}
$$

For aesthetic reasons, we choose vacuum as well as physical states of the theory to be the harmonic states in the Hodge decomposition theorem because they are BRST- and co-BRST invariant together (i.e. $Q_{(B, D)}\left|v a c>=0, Q_{(B, D)}\right|$ phys $>=0$ ). This will also ensure that there are no energy excitations in the theory because the VEV (i.e. $<v a c\left|\hat{\widetilde{T}}_{00}\right| v a c>=0$ ) as well as the excitations in the physical states (i.e. $<$ phys $\left|\hat{T}_{00}\right| p h y s^{\prime}>=0$ ) turns out to be zero. This result reconfirms the topological nature of the theory under discussion.

Besides BRST- and co-BRST charges, there are anti- BRST and anti-dual BRST charges in the theory which are also nilpotent of order two. For the non-Abelian gauge theories, the corresponding symmetries can be obtained only by introducing some auxiliary fields. The ensuing modified Lagrangian densities (which are equivalent extensions of (3.1)) are

$$
\begin{aligned}
& \mathcal{L}_{\mathcal{B}}=\mathcal{B}^{a} E^{a}-\frac{1}{2} \mathcal{B}^{a} \mathcal{B}^{a}+B^{a}(\partial \cdot A)^{a}+\frac{1}{2}\left(B^{a} B^{a}+\bar{B}^{a} \bar{B}^{a}\right)-i \partial_{\mu} \bar{C}^{a} D^{\mu} C^{a} \\
& \mathcal{L}_{\bar{B}}=\mathcal{B}^{a} E^{a}-\frac{1}{2} \mathcal{B}^{a} \mathcal{B}^{a}-\bar{B}^{a}(\partial \cdot A)^{a}+\frac{1}{2}\left(B^{a} B^{a}+\bar{B}^{a} \bar{B}^{a}\right)-i D_{\mu} \bar{C}^{a} \partial^{\mu} C^{a}
\end{aligned}
$$

where $\mathcal{B}^{a}, B^{a}$ and $\bar{B}^{a}$ are the auxiliary fields. The latter two are restricted to satisfy the following relation $[34]$

$$
B^{a}+\bar{B}^{a}=i g f^{a b c} C^{b} \bar{C}^{c} .
$$


The following off-shell nilpotent $\left(\delta_{A D}^{2}=0, \delta_{A B}^{2}=0\right)$ anti-BRST $\left(\delta_{A B}\right)$ and anti-dual BRST $\left(\delta_{A D}\right)$ symmetry transformations

$$
\begin{aligned}
& \delta_{A B} A_{\mu}^{a}=\eta D_{\mu} \bar{C}^{a} \quad \delta_{A B} \bar{C}^{a}=-\frac{\eta g}{2} f^{a b c} \bar{C}^{b} \bar{C}^{c} \quad \delta_{A B} C^{a}=i \eta \bar{B}^{a} \quad \delta_{A B} \bar{B}^{a}=0 \\
& \delta_{A B} B^{a}=\eta g f^{a b c} B^{b} \bar{C}^{c} \quad \delta_{A B} \mathcal{B}^{a}=\eta g f^{a b c} \mathcal{B}^{b} \bar{C}^{c} \quad \delta_{A B} E^{a}=\eta g f^{a b c} E^{b} \bar{C}^{c} \\
& \delta_{A D} A_{\mu}^{a}=-\eta \varepsilon_{\mu \nu} \partial^{\nu} C^{a} \quad \delta_{A D} C^{a}=0 \quad \delta_{A D} \bar{C}^{a}=i \eta \mathcal{B}^{a} \quad \delta_{A D} \bar{B}^{a}=0 \\
& \delta_{A D} B^{a}=0 \quad \delta_{A D} \mathcal{B}^{a}=0 \quad \delta_{A D} E^{a}=\eta D_{\mu} \partial^{\mu} C^{a} \quad \delta_{A D}(\partial \cdot A)^{a}=0
\end{aligned}
$$

leave (3.13b) quasi invariant as: $\delta_{A B} \mathcal{L}_{\bar{B}}=-\eta \partial_{\mu}\left[\bar{B}^{a} D^{\mu} \bar{C}^{a}\right], \delta_{A D} \mathcal{L}_{\bar{B}}=\eta \partial_{\mu}\left[\mathcal{B}^{a} \partial^{\mu} C^{a}\right]$. These symmetry transformations are generated by the following conserved charges

$$
\begin{aligned}
Q_{A B} & =\int d x\left[\dot{\bar{B}}^{a} \bar{C}^{a}-\bar{B}^{a} D_{0} \bar{C}^{a}-\frac{i g}{2} f^{a b c} \dot{C}^{a} \bar{C}^{b} \bar{C}^{c}\right] \\
Q_{A D} & =\int d x\left[\mathcal{B}^{a} \dot{C}^{a}-D_{0} \mathcal{B}^{a} C^{a}-i g f^{a b c} C^{a} \partial_{1} C^{b} \bar{C}^{c}\right] .
\end{aligned}
$$

These charges further extend the BRST algebra (3.7) as given below

$$
\begin{aligned}
& Q_{A B}^{2}=0 \quad Q_{A D}^{2}=0 \quad\left[W^{(N)}, Q_{A B}\right]=\left[W^{(N)}, Q_{A D}\right]=0 \quad\left\{Q_{D}, Q_{A B}\right\}=0 \\
& \left\{Q_{A B}, Q_{A D}\right\}=W^{(N)} \quad\left\{Q_{B}, Q_{A B}\right\}=0 \quad\left\{Q_{D}, Q_{A D}\right\}=0 \\
& \left\{Q_{B}, Q_{A D}\right\}=0 \quad i\left[Q_{G}, Q_{A B}\right]=-Q_{A B} \quad i\left[Q_{G}, Q_{A D}\right]=Q_{A D} .
\end{aligned}
$$

This algebra is also analogous to the algebra satisfied by the de Rham cohomology operators (2.9a). Thus, we notice that the set $\left(Q_{A D}, Q_{A B}, W^{(N)}\right)$ can also be identified with the set of cohomological operators $(d, \delta, \Delta)$ defined on a compact manifold.

It is obvious that we have four conserved and nilpotent charges in the theory. On a $2 \mathrm{D}$ compact manifold, topological invariants for the non-Abelian gauge theory can be defined analogous to the Abelian gauge theory (cf. (2.20)) by replacing $V_{k}$ and $W_{k}$ by $B_{k}, D_{k}$ and $A_{k}^{(b)}, A_{k}^{(d)}$. In fact, we can obtain a set of three topological invariants $\left(B_{k}, k=0,1,2\right)$ w.r.t.

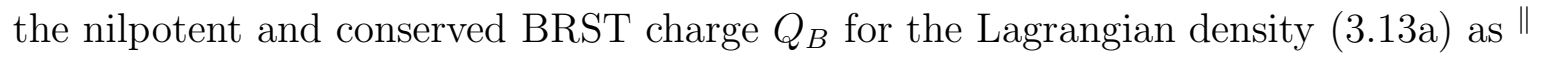

$$
\begin{aligned}
& B_{0}=B^{a} C^{a}-\frac{i g}{2} f^{a b c} \bar{C}^{a} C^{b} C^{c} \\
& B_{1}=\left[B^{a} A_{\mu}^{a}+i C^{a} D_{\mu} \bar{C}^{a}\right] d x^{\mu} \\
& B_{2}=i\left[A_{\mu}^{a} D_{\nu} \bar{C}^{a}-\bar{C}^{a} D_{\mu} A_{\nu}^{a}\right] d x^{\mu} \wedge d x^{\nu} .
\end{aligned}
$$

Similarly, the set of three topological invariants $\left(D_{k}, k=0,1,2\right)$ on the 2D compact manifold, defined w.r.t. dual BRST charge $Q_{D}$, is

$$
\begin{aligned}
& D_{0}=\mathcal{B}^{a} \bar{C}^{a} \\
& D_{1}=\left[\bar{C}^{a} \varepsilon_{\mu \rho} \partial^{\rho} C^{a}-i \mathcal{B}^{a} A_{\mu}^{a}\right] d x^{\mu} \\
& D_{2}=i\left[\varepsilon_{\mu \rho} \partial^{\rho} C^{a} A_{\nu}^{a}+\frac{1}{2} C^{a} \varepsilon_{\mu \nu}(\partial \cdot A)^{a}\right] d x^{\mu} \wedge d x^{\nu} .
\end{aligned}
$$

The definition of the anti- BRST charge $\left(Q_{A B}\right)$ in non-Abelian gauge theory is more involved as we introduce some new auxiliary fields. In terms of these auxiliary fields and the basic

\footnotetext{
" Note that, in addition to the transformations in (3.2), there are some off-shell nilpotent BRST transformations: $\delta_{B} \bar{C}^{a}=i \eta B^{a} \delta_{B} B^{a}=0 \delta_{B} \mathcal{B}^{a}=\eta g f^{a b c} \mathcal{B}^{b} C^{c} \delta_{B} \bar{B}^{a}=\eta g f^{a b c} \bar{B}^{b} C^{c}$ for (3.13a).
} 
fields, the three invariants $\left(A_{k}^{(b)}, k=0,1,2\right)$ corresponding to zero-, one- and two-forms are

$$
\begin{aligned}
& A_{0}^{(b)}=\bar{B}^{a} \bar{C}^{a}-\frac{i g}{2} f^{a b c} C^{a} \bar{C}^{b} \bar{C}^{c} \\
& A_{1}^{(b)}=\left[\bar{B}^{a} A_{\mu}^{a}+i \bar{C}^{a} D_{\mu} C^{a}\right] d x^{\mu} \\
& A_{2}^{(b)}=i\left[A_{\mu}^{a} D_{\nu} C^{a}-C^{a} D_{\mu} A_{\nu}^{a}\right] d x^{\mu} \wedge d x^{\nu} .
\end{aligned}
$$

Finally, three invariants $\left(A_{k}^{(d)}, k=0,1,2\right)$, corresponding to the anti-dual BRST charge $\left(Q_{A D}\right)$, are

$$
\begin{aligned}
& A_{0}^{(d)}=\mathcal{B}^{a} C^{a} \\
& A_{1}^{(d)}=\left[C^{a}\left(\varepsilon_{\mu \rho} \partial^{\rho} \bar{C}^{a}\right)-i \mathcal{B}^{a} A_{\mu}^{a}\right] d x^{\mu} \\
& A_{2}^{(d)}=i\left[\varepsilon_{\mu \rho} \partial^{\rho} \bar{C}^{a} A_{\nu}^{a}+\frac{1}{2} \bar{C}^{a} \varepsilon_{\mu \nu}(\partial \cdot A)^{a}\right] d x^{\mu} \wedge d x^{\nu} .
\end{aligned}
$$

These topological invariants obey the same type of recursion relations as are expected of the topological invariants of a well-defined TFT. These relations are $[2,4,35]$

$$
\begin{array}{lll}
\delta_{B} B_{k}=\eta d B_{k-1} & \delta_{A B} A_{k}^{(b)}=\eta d A_{k-1}^{(b)} & d=d x^{\mu} \partial_{\mu} \\
\delta_{D} D_{k}=\eta \delta D_{k-1} & \delta_{A D} A_{k-1}^{(d)}=\eta \delta A_{k}^{(d)} & \delta=i d x^{\mu} \varepsilon_{\mu \nu} \partial^{\nu} .
\end{array}
$$

These properties establish the topological nature of 2D self-interacting non-Abelian gauge theory. This theory belongs to a new class of TFT as is evident from its differences with Witten- and Schwarz type of TFTs.

As was emphasized at the end of equation (2.25), one can obtain an analogue of the (*) operation from the requirement that the topological invariants of the self-interacting non-Abelian gauge theory should obey the same kind of relations as (2.24) and (2.25) for the Abelian gauge theory. It can be checked that under the following transformations

$$
\begin{aligned}
& C^{a} \rightarrow i \bar{C}^{a} \quad \bar{C}^{a} \rightarrow i C^{a} \quad A_{\mu}^{a} \rightarrow A_{\mu}^{a} \\
& B^{a} \rightarrow-i \mathcal{B}^{a}-\frac{i g}{2} f^{a b c} C^{b} \bar{C}^{c} \quad \mathcal{B}^{a} \rightarrow-i B^{a}-\frac{g}{2} f^{a b c} C^{b} \bar{C}^{c} \\
& \partial_{\mu} \delta^{a b} \rightarrow i \varepsilon_{\mu \rho} \partial^{\rho} \delta^{a b}+\frac{g}{2} f^{a b c} A_{\mu}^{c} \quad \varepsilon_{\mu \rho} \partial^{\rho} \delta^{a b} \rightarrow i \partial_{\mu} \delta^{a b}-\frac{i g}{2} f^{a b c} A_{\mu}^{c}
\end{aligned}
$$

the topological invariants, for $k=0,1,2$, transform as

$$
B_{k} \rightarrow D_{k} \quad A_{k}^{(b)} \rightarrow A_{k}^{(d)} \quad D_{k} \rightarrow(-1)^{k} B_{k} \quad A_{k}^{(d)} \rightarrow(-1)^{k} A_{k}^{(b)} .
$$

In the proof of the above relations, we should include in (3.23)

$$
\begin{aligned}
& \mathcal{B}^{a} \rightarrow-i \bar{B}^{a}-\frac{g}{2} f^{a b c} \bar{C}^{b} C^{c} \\
& \bar{B}^{a} \rightarrow-i \mathcal{B}^{a}-\frac{i g}{2} f^{a b c} \bar{C}^{b} C^{c}
\end{aligned}
$$

for the checking of the transformation properties of topological invariants w.r.t. $Q_{A B}$ and $Q_{A D}$. In the language of the above $(*)$ operation, the relations (3.24) can be mathematically expressed as

$$
* B_{k}=D_{k} \quad * A_{k}^{(b)}=A_{k}^{(d)} \quad *\left(* B_{k}\right)=(-1)^{k} B_{k} \quad *\left(* A_{k}^{(b)}\right)=(-1)^{k} A_{k}^{(b)}
$$

It will be noticed that $(3.24,3.26))$ are exactly like $(2.24,2.25)$. However, the transformations $(3.23,3.25)$ are still not the analogue of the exact Hodge dual $(*)$ operation of 
differential geometry. Unlike the case of 2D Abelian gauge theory where this analogy was perfect, we see that, for the non-Abelian case, the above transformations do not keep the Lagrangian density $(3.1)$ or $(3.13 \mathrm{a}, \mathrm{b})$ invariant. Furthermore, the dual BRST transformation $\delta_{D}$ can not be obtained from BRST transformations $\delta_{B}$ by exploiting (3.23). In addition, the analogues of (2.14) and (2.16) do not exist.

We conclude this section with the remark that self-interacting $2 \mathrm{D}$ non-Abelian gauge theory is a topological field theory which bears the appearance of a Witten type TFT but possesses symmetries that are reminiscent of a Schwarz type of TFT.

\section{Summary and discussion}

It has been shown that free Abelian- and self-interacting non-Abelian gauge theories in 2D belong to a new class of topological field theories. The ideas of BRST cohomology and Hodge decomposition theorem play a pivotal role in the proof of topological nature of these theories. The local symmetries of these theories define the de Rham cohomology operators and a discrete symmetry transformation corresponds to the Hodge $(*)$ operation of differential geometry. As far as these symmetries are concerned, there are some specific differences as well as similarities between 2D free Abelian- and self-interacting ** nonAbelian gauge theories. For instance, the curvature tensor for the Abelian gauge theory is derived from $F^{(A)}=d A$ when $d$ directly operates on the one-form $A$. This is not the case with the non-Abelian gauge theory where $F^{(N)}=(d+A) \wedge A$. Under the BRST transformations, however, the kinetic energy terms for Abelian- and non-Abelian gauge theories do remain invariant even though $\delta_{b} F_{\mu \nu}^{(A)}=0$ but $\delta_{B} F_{\mu \nu}^{a(N)}=\eta g f^{a b c} F_{\mu \nu}^{b(N)} C^{c}$. Under the dual BRST symmetries, the gauge-fixing terms of both the theories remain invariant as they are obtained by the application of $\delta= \pm * d *$ operator on the one-form $A$. The Casimir operator generates a symmetry transformation in which ghost fields do not transform and $A_{\mu}$ gauge field transforms to its own equation of motion in both the cases (cf. (2.4), (3.3)).

It is interesting to note that, in the Abelian gauge theory, the requirement that the topological invariants should be related with each-other $\left(I_{k} \rightarrow J_{k}\right)$ by a Hodge $(*)$ operation, singles out (2.10) from the set of transformations (2.10) and (2.11). On the other hand, it can be seen that for the interacting 2D Abelian gauge theory where Abelian gauge field $A_{\mu}$ couples with the Dirac fields, it is (2.11) that is singled out for the generalization to include matter (Dirac) fields [36] and (2.10) is ruled out for such an important extension. Furthermore, it is gratifying to note that certain specific transformation properties of the topological invariants (cf. (2.24), (2.25) (3.24), (3.26)) lead to the derivation of transformations (3.23) for the non-Abelian gauge theory which reduce to the Abelian case (2.10) under the limit $g \rightarrow 0$. In fact, it seems to us, the root cause of the lack of a perfect definition of Hodge $(*)$ operation for the non-Abelian gauge theory, in the language of symmetry prop-

** Besides interaction among themselves, the non-Abelian gauge fields also interact with ghost fields. The latter fields are, however, not the physical matter fields. 
erty, is the difference in the definition of a non-Abelian curvature tensor $F^{(N)}=(d+A) \wedge A$ from that of an Abelian gauge theory where $F^{(A)}=d A$. The latter is a perfect Hodgeas well as topological field theory. In fact, for the non-Abelian gauge theories, it can be seen that the set $(d+A, \delta, \Delta)$ does not define the perfect de Rham cohomological operators whereas for the Abelian gauge theory the set $(d, \delta, \Delta)$ does define the same.

In some of our works [23-25,32] (including the present one), we have carried out investigations in the context of BRST cohomology and HDT in 2D and 4D where spacetime is endowed with a flat Minkowski metric. The topological nature emerges due to the fact that there are no propagating degrees of freedom associated with the gauge bosons. It would be an interesting endeavour to consider the interacting gauge theories where matter fields are also present. Some steps in this direction [36,37] have already been taken. It appears to us, at the moment, that the BRST cohomology and HDT would shed some light on the Adler-Bardeen-Jackiw (ABJ) anomaly in 2D where a $U(1)$ gauge field is coupled to the conserved current of the Dirac fields. For such an interacting theory, it has been shown that the dual BRST symmetry is connected with the chiral $\left(\gamma_{5}\right)$ transformation on the matter (Dirac) fields [36,37]. It would be nice to generalize this assertion to the non-Abelian gauge theory in 2D where there is an interaction with matter (Dirac) fields.

The central outcome of of our studies of the BRST cohomology and HDT should be taken as the proof of the existence of a new class of topological field theories which are nothing but the free 2D Abelian- and self-interacting non-Abelian gauge theories. Furthermore, we strongly feel that our studies would shed some light on the consistency and unitarity of the anomalous gauge theories in 2D (see, e.g., Refs. [38,39] and references therein). These studies might provide an insight to study thoroughly $(3+1)$-dimensional TFTs in the framework of BRST cohomology and HDT which will have something to do with the real spacetime manifolds.

The key results in the study of TFTs with a nontrivial spacetime metric has been the classification of 3D and 4D manifolds which have been of importance in the context of string theories $[2,4,6,7]$. It would be a nice future direction to study $2 \mathrm{D}$ and $4 \mathrm{D}$ theories [23-25,32] with a nontrivial spacetime metric in the framework of BRST cohomology and HDT and explore the outcome of such studies. In fact, we guess that the results of earlier works $[23-25,32]$ can be generalized to the case of nontrivial metrics. For this to be true, one has to show the metric independence of the path integral measure. In Ref. [35], it has been established that the existence of a BRST type fermionic-bosonic symmetry is good enough to prove the metric independence of the measure. It is obvious that, we have such kind of BRST- and co-BRST symmetries in our theories. Thus, heuristically, it appears to us that the measure will be independent of the choice of the metric for these theories as well. After this, it will be straightforward to show that the partition functions as well as the expectation values of the BRST-, co-BRST- and topological invariants are metric independent. To demonstrate this, one has to require that: $Q_{B} \mid$ phys $>=0, Q_{D} \mid$ phys $>=0$ (see, e.g., Ref. [12]). This requirement, very clearly, entails on the physical states of the 
theory to be the harmonic state of the HDT. These are some of the issues which are under investigation and our results will be reported elsewhere [40].

\section{References}

[1] Schwarz A S 1978 Lett. Math. Phys. 2247

[2] Witten E 1982 J. Diff. Geom. 17661 Witten E 1982 Nucl. Phys. B202 253

[3] Witten E 1988 Commun. Math. Phys. 117353

Witten E 1989 Commun. Math. Phys. 121351

[4] Distler J 1990 Nucl. Phys. B342 523

[5] Witten E 1988 Phys. Lett. 206601

[6] Witten E 1988 Nucl. Phys. B311 46 Witten E 1990 Nucl. Phys. B340 281

[7] Labastida J Pernici M and Witten E 1988 Nucl. Phys. B310 611

[8] Donaldson S 1983 J. Diff. Geom. 18279 Donaldson S 1990 Topology 29257

[9] Jones V 1985 Bull. Am. Math. Soc. 12103

[10] Baulieu L and Singer I M 1988 Nucl. Phys. (Proc. Suppl.) 5B 12

[11] Floer A 1989 J. Diff. Geom. 30207 Floer A 1988 Commun. Math. Phys. 118215

[12] For review, see, e.g., Birmingham D, Blau M, Rakowski M and Thompson G 1991 Phys. Rep. 209129

[13] Eguchi T, Gilkey P B and Hanson A J 1980 Phys. Rep. 66213

[14] Mukhi S and Mukunda N 1990 Introduction to Topology, Differential Geometry and Group Theory for Physicists (New Delhi: Wiley Eastren)

[15] Nishijima K 1988 Prog. Theor. Phys. 80897 Nishijima K 1988 Prog. Theor. Phys. 80905

[16] van Holten J W 1990 Phys. Rev. Lett. 642863 van Holten J W 1990 Nucl. Phys. B339 158 Aratyn H 1990 J. Math. Phys. 311240

[17] Fülöp G and Marnelius R 1995 Nucl. Phys. B456 442 Kalau W and van Holten J W 1991 Nucl. Phys. B361 233

[18] McMullan D and Lavelle M 1993 Phys. Rev. Lett. 713758 McMullan D and Lavelle M 1995 Phys. Rev. Lett. 754151

[19] Rivelles V O 1995 Phys. Rev. Lett. 754150 Rivelles V O 1996 Phys. Rev. D53 3257 
[20] Marnelius R 1997 Nucl. Phys. B494 346

[21] Yang H S and Lee B -H 1996 J. Math. Phys. 376106

[22] Zhong T and Finkelstein D 1994 Phys. Rev. Lett. 733055 Zhong $\mathrm{T}$ and Finkelstein D $1995 \mathbf{7 5} 4152$

[23] Malik R P 2000 Int. J. Mod. Phys. A15 1685 : hep-th/9808040

[24] Malik R P 2000 J. Phys. A: Math Gen 33 2437: hep-th/9902146

[25] Malik R P 1999 Mod. Phys. Lett. A14 1937 : hep-th/9903121

[26] Nishijima K 1986 in: Progress in Quantum Field Theory Eds. Ezawa H and Kamefuchi S (Amsterdam: North-Holland)

[27] Henneaux M and Teitelboim C 1992 Quantization of Gauge Systems (Princeton NJ: Princeton University Press)

[28] Nakanishi N and Ojima I 1990 Covariant Operator Formalism of Gauge Theories and Quantum Gravity (Singapore: World Scientific)

[29] Deser S, Gomberoff A, Henneaux M and Teitelboim C 1997 Phys. Lett. B400 80

[30] Banerjee R and Wotzasek C 1998 Nucl. Phys. B527 402

[31] Banerjee R and Chakraborty B 1999 J. Phys. A: Math Gen 324441

[32] Harikumar E, Malik R P and Sivakumar M 2000 J. Phys. A: Math Gen 337149 : hep-th/0004145

[33] Weinberg S 1996 The quantum theory of fields: Modern applications (Cambridge: Cambridge University Press) Vol. II

[34] Curci G and Ferrari R 1976 Phys. Lett. B63 51

Bonora L and Tonin M 1981 Phys. Lett. B98 48

[35] Kaul R K and Rajaraman R 1991 Phys. Lett. B265 335

Kaul R K and Rajaraman R 1990 Phys. Lett. B249 433

[36] Malik R P Dual BRST symmetry for two dimensional QED Preprint hep-th/9711056

[37] Malik R P 2000 Mod. Phys. Lett A15 2079 : hep-th/0003128

[38] Jackiw R and Rajaraman R 1985 Phys. Rev. Lett. 541219

[39] Malik R P 1988 Phys. Lett. B212 445

[40] Malik R P (in preparation) 